\title{
Mathematical Methods Applied to Economy Optimization of an Electric Vehicle with Distributed Power Train System
}

\author{
Binbin Sun, Song Gao, and Chao Ma \\ School of Transportation and Vehicle Engineering, Shandong University of Technology, Zibo, Shandong 255049, China \\ Correspondence should be addressed to Song Gao; gaos546@126.com
}

Received 21 December 2015; Accepted 31 January 2016

Academic Editor: Rafael Toledo

Copyright (C) 2016 Binbin Sun et al. This is an open access article distributed under the Creative Commons Attribution License, which permits unrestricted use, distribution, and reproduction in any medium, provided the original work is properly cited.

\begin{abstract}
This research presents mathematical methods to develop a high-efficiency power train system for a microelectric vehicle (MEV). First of all, to get the optimal ratios of a two-speed gearbox, the functional relationship of energy consumption and transmissions is established using the design of experiment (DOE) and min-max fitting distance methods. The convex characteristic of the model and the main and interactive effects of transmissions on energy consumption are revealed and hill-climbing method is adopted to search the optimal ratios. Then, to develop an efficient and real-time drive strategy, an optimization program is proposed including shift schedule, switch law, and power distribution optimization. Particularly, to construct a mathematical predictive distribution model, firstly Latin hypercube design (LHD) method is adopted to generate random and discrete operations of the MEV; secondly the optimal power distribution coefficients under various LHD points are confirmed based on offline genetic algorithm (GA); then Gauss radial basis function (RBF) is utilized to solve the low-precision problem in polynomial model. Finally, simulation verifications of the optimized scheme are carried out. Results show that the proposed mathematical methods for the optimizations of transmissions and drive strategy are able to establish a high-efficiency power train system.
\end{abstract}

\section{Introduction}

Facing the challenges of world fossil energy crisis and environmental pollution, great efforts have been made to develop various motor drive train systems to substitute internal combustion engine [1]. MEV, since the advantage of lightweight body, has higher energy efficiency and lower requirement for battery capacity. Therefore, pending the solutions of high cost and low specific capacity of battery, MEV shares a higher market maintenance in many countries $[2,3]$.

Due to the limited layout space, drive train configuration with single motor and settled transmission (SMST) is the most commonly used scheme in MEV [4]. In order to meet the design requirements of acceleration and maximum speed in electric vehicle industry, motor with higher power is required in this configuration; however it may lead MEV working under low-load conditions frequently and thus cause negative influence on vehicle economy. Therefore, great efforts have been made to develop feasible and energy-saving drive schemes for MEV.
In-wheel motor driving configuration [5], acquiring small layout space and simultaneously showing good economy performance, has attracted a great deal of attention. Plentiful achievements have been obtained on dynamics and structure optimization, integrated control, and speed differential control [6]. But as the challenges of system reliability and stability are still unsettled [7], it is still quite difficult to popularize engineering application of this technology.

Configuration with single motor and two-speed transmissions (SMTST) $[8,9]$ offers potential for system efficiency improvement owing to the controllable gear ratio. Studies on gear train design, gear shift control, and ratio optimization have been carried out [10-12]. Results have confirmed the benefits of the SMTST scheme on economy improvement. But there is still much more potential to be excavated in this configuration for system efficiency optimization by using distributed scheme [13].

In consideration of both the layout space and system reliability of MEV, based on the traditional distributed drive train configuration [14-17], a special power train system is 


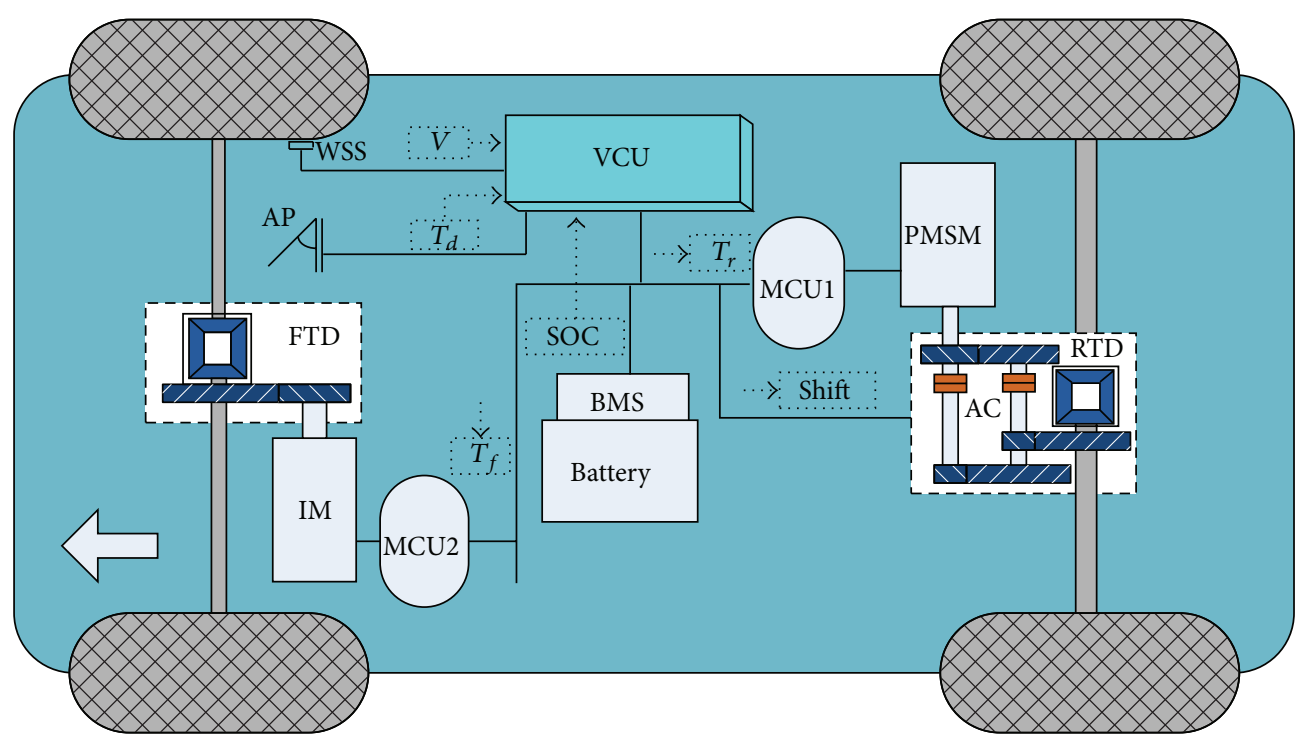

Figure 1: Power train system of the DMVT-EV.

proposed in this paper, which consists of a small permanent magnetic synchronous motor (PMSM) with two-speed transmission in rear shaft and a high power induction motor (IM) with one-speed gearbox in front shaft. For this dualmotor-and-variable-transmission (DMVT) configuration, to seek the optimal gear ratios and drive strategy are the keys to achieving high-efficiency operation of the MEV.

Currently, intelligent algorithms, such as dynamic programming (DP) and GA, are the most common methods to optimize transmissions $[4,18]$. These methods mainly concentrate on the optimal solution while neglecting the recessive relationship of transmissions and EV economy. As for power distribution optimization for the multimotor drive train system, strategy based on unchangeable or fuzzy rule is an effective method to achieve real-time control but has a relatively low-efficiency problem in the meantime [19-21]. On the contrary, intelligent optimizations are capable of finding the optimal result, while they cannot meet the requirement of real-time control [22-24]. Moreover, for the DMVT scheme, there is still a lack of optimum synthesis of the entire drive system at present, where power distribution strategy may interact with shift schedule and switch law of the single high or single small motor drive mode (SHMDM or SSMDM).

This paper therefore proposes mathematical methods for optimizing the DMVT system. On one hand, different from the traditional methods, a predictive model of energy consumption $\left(E_{\mathrm{dis}}\right)$ as function of gear ratios $\left(i_{1}, i_{2}\right)$ is proposed. To establish this mathematical model, firstly, design of experiment (DOE) is used for the level design of ratios. Secondly, based on the DOE points, the numerical model of $E_{\text {dis }}$ is created aiming at the best drive efficiency. Then, min-max distance method is suggested instead of the traditional least squares method. Finally, the characteristic of the mathematical model and the main and interactive effects of transmissions on $E_{\text {dis }}$ are researched and hill-climbing method is utilized to search the best $i_{1}$ and $i_{2}$.
On the other hand, in order to create a real-time and efficient drive strategy, an offline optimization program with multiple subroutines is designed. Particularly, in contrast with the traditional rule-based distribution method, a mathematical predictive distribution model is proposed. Firstly, to meet the random characteristic of the MEV operations, Latin hypercube design (LHD) is adopted to generate experimental combinations of vehicle velocity $(V)$, power demand $\left(P_{d}\right)$, and state of charge (SOC). Then, an offline GA with penalty function is adopted to search the optimal distribution coefficients under the various LHD points, aiming at the least energy consumption of battery while taking overload punishment in consideration. In addition, to ensure the predictive precision, Gauss radial basis function (RBF) is used to fit the optimal data.

Finally, the optimized scheme based on mathematical methods is discussed and verified by simulation method in J1015 drive cycle, which is used for vehicle test under unban cycle conditions.

\section{Configuration of the MEV}

As shown in Figure 1, the distributed power train configuration offers great flexibility and potential for optimized design. Based on the sampling signals, which are $V$ from wheel speed sensor (WSS), torque demand $\left(T_{d}\right)$ from acceleration pedal (AP), and SOC from battery management system (BMS), vehicle control unit (VCU) makes strategic decisions to achieve shift control, and torque distribution for the front and rear transmission and differential systems (FTD and RTD). Motor control units 1 and 2 (MCU1 and MCU2) are utilized to control the rear and front motor independently. The two automatic clutches (AC) in rear shaft are used to control shift.

As the marked characteristics of unban drive conditions are low vehicle velocity and low workload, a small power PMSM is deposed in rear shaft to take advantage of the PMSM 
TABLE 1: Motor performance for the DMVT-EV.

\begin{tabular}{lcc}
\hline Items & Small power motor & High power motor \\
\hline Nominal power & $10 \mathrm{~kW}$ & $15 \mathrm{~kW}$ \\
Pear power & $20 \mathrm{~kW}$ & $30 \mathrm{~kW}$ \\
Nominal speed & $3200 \mathrm{r} / \mathrm{min}$ & $3000 \mathrm{r} / \mathrm{min}$ \\
Peak speed & $7000 \mathrm{r} / \mathrm{min}$ & $6500 \mathrm{r} / \mathrm{min}$ \\
Peak torque & 65 & 95.5 \\
Motor type & PMSM & IM \\
\hline
\end{tabular}

efficiency characteristic that can generate torque efficiently in low-speed regions. Furthermore, a two-speed gearbox is attached to the PMSM to expand the high- efficiencyworking-condition scope. A high power IM with one-speed gearbox is equipped on the front-wheel side to make use of the IM efficiency characteristic that can generate torque efficiently at high speed. On the other hand, high power motor can maintain normal running of the MEV under overload conditions. The specific parameters of the two motors are presented in Table 1.

As shown in Table 2, according to the MEV velocity and power demand, this configuration can provide five driving modes, which increases the availability of efficient operations in unban drive cycle. However, the optimal ratios of the two-speed gearbox and the efficient and real-time drive strategy are still not confirmed. Optimized design based on mathematical methods for the 1st and 2nd gear ratio and driving strategy are the keys in this study.

\section{The Mathematical Model of Energy Consumption}

Figure 2 shows the route for establishing the mathematical predictive model of energy consumption $\left(E_{\mathrm{dis}}\right)$ as function of transmissions $\left(i_{1}, i_{2}\right)$. The design process consists of variables design using the method of DOE, problems formulation aiming at the least energy consumption, and modelling of the energy consumption based on min-max fitting distance method.

3.1. Variables Design. First of all, the first shift ratio $\left(i_{1}\right)$ of the small power drive train system should meet part of the peak torque demand on the maximum ascendable road grade, as shown in the following formula:

$$
i_{1} \geq k \frac{r\left(G f \cos \alpha_{\max }+G \sin \alpha_{\max }+C_{D} A V^{2} / 21.15\right)}{T_{s \max }},
$$

where $k$ is the proportion of the maximum torque demand that the small power motor should provide; $r$ represents wheel radius; $G$ is the total weight of the MEV; $f$ is the coefficient of rolling resistance; $\alpha_{\max }$ is the maximum grade angle; $V$ is vehicle velocity; $T_{\text {smax }}$ is the peak torque of the small power motor; $C_{D}$ is coefficient of air resistance; $A$ is the area of the MEV section surface.
Secondly, in order to satisfy the maximum velocity of the MEV, the second shift ratio $\left(i_{2}\right)$ of the small power drive train should be subject to the following inequality constraint:

$$
i_{2} \leq \frac{0.377 r N_{s \max }}{V_{\max }},
$$

where $V_{\max }$ means the maximum velocity of the MEV and $N_{\text {smax }}$ is the peak speed of the small power motor.

Finally, as it is unpractical to obtain the effect of every possible gear ratio combination on the battery energy consumption, LDH method is used to design as few experiment points as possible while it can represent the characteristic of the possible combinations according to the constrains of $i_{1}$ and $i_{2}$ proposed above. The designed points based on LHD are shown in the following formula:

$$
\vec{X}=\left[\begin{array}{cc}
i_{11} & i_{21} \\
\vdots & \vdots \\
i_{1 k} & i_{2 k} \\
\vdots & \vdots \\
i_{1 m} & i_{2 m}
\end{array}\right]=\left[\begin{array}{cc}
8.9784 & 4.4475 \\
\vdots & \vdots \\
12.0863 & 4.5745 \\
\vdots & \vdots \\
12.0216 & 5.0824
\end{array}\right] .
$$

3.2. Problem Formulations. First of all, as the small power drive system is designed to achieve higher vehicle economy under low-to-middle velocity and workload conditions, the sampling intervals of $V$ and $T_{d}$ are constrained by $0-45 \mathrm{~km} / \mathrm{h}$ and $0-200 \mathrm{~N} \cdot \mathrm{m}$, respectively. Then, according to (4), the wheel torque demand and vehicle power demand under J1015 drive cycle can be confirmed as follows:

$$
\begin{aligned}
T_{d j} & =\left(G f+\frac{C_{D} A V_{j}^{2}}{21.15}+\delta m \frac{\left(V_{j}-V_{j-1}\right)}{\Delta t}\right) r, \\
P_{d j} & =\frac{T_{d j} V_{j}}{3600 r},
\end{aligned}
$$

where $T_{d j}$ is the wheel torque demand under the number $j$ sampling point of the J1015 cycle; $\Delta t$ is the sampling period; $m$ is the vehicle mass; $\delta$ is the rotational inertia coefficient; $P_{d j}$ is the vehicle power demand under the number $j$ sampling point of the J1015 cycle.

Secondly, for the number $k$ LHD point, according to mechanical system equations, the motor rotate speed, torque, and efficiency under the number $j$ cycle condition are obtained as follows:

$$
\begin{aligned}
N_{1 \text { st } j k} & =\frac{i_{1 k} V_{j}}{0.377 r}, \\
N_{2 \text { nd_ } j k} & =\frac{i_{2 k} V_{j}}{0.377 r}, \\
T_{1 \text { st_ } j k} & =\frac{T_{d j}}{\eta_{T} i_{1 k}}, \\
T_{2 \text { nd_ } j k} & =\frac{T_{d j}}{\eta_{T} i_{2 k}},
\end{aligned}
$$


TABLE 2: Available drive modes of the DMVT-EV.

\begin{tabular}{|c|c|c|c|}
\hline Drive mode & Front power train & Rear power train & Application \\
\hline 1 & Motor off & $\begin{array}{l}\text { Motor on } \\
+1 s t \text { shift }\end{array}$ & Low velocity, middle load \\
\hline 2 & Motor off & $\begin{array}{l}\text { Motor on } \\
+2 \text { nd shift }\end{array}$ & Middle velocity, low load \\
\hline 3 & Motor on & Off & Middle-to-high velocity, middle load \\
\hline 4 & Motor on & $\begin{array}{l}\text { Motor on } \\
+1 \text { st shift }\end{array}$ & Low-to-middle velocity, high load \\
\hline 5 & Motor on & $\begin{array}{l}\text { Motor on } \\
+2 \text { nd shift }\end{array}$ & Middle-to-high velocity, high load \\
\hline
\end{tabular}

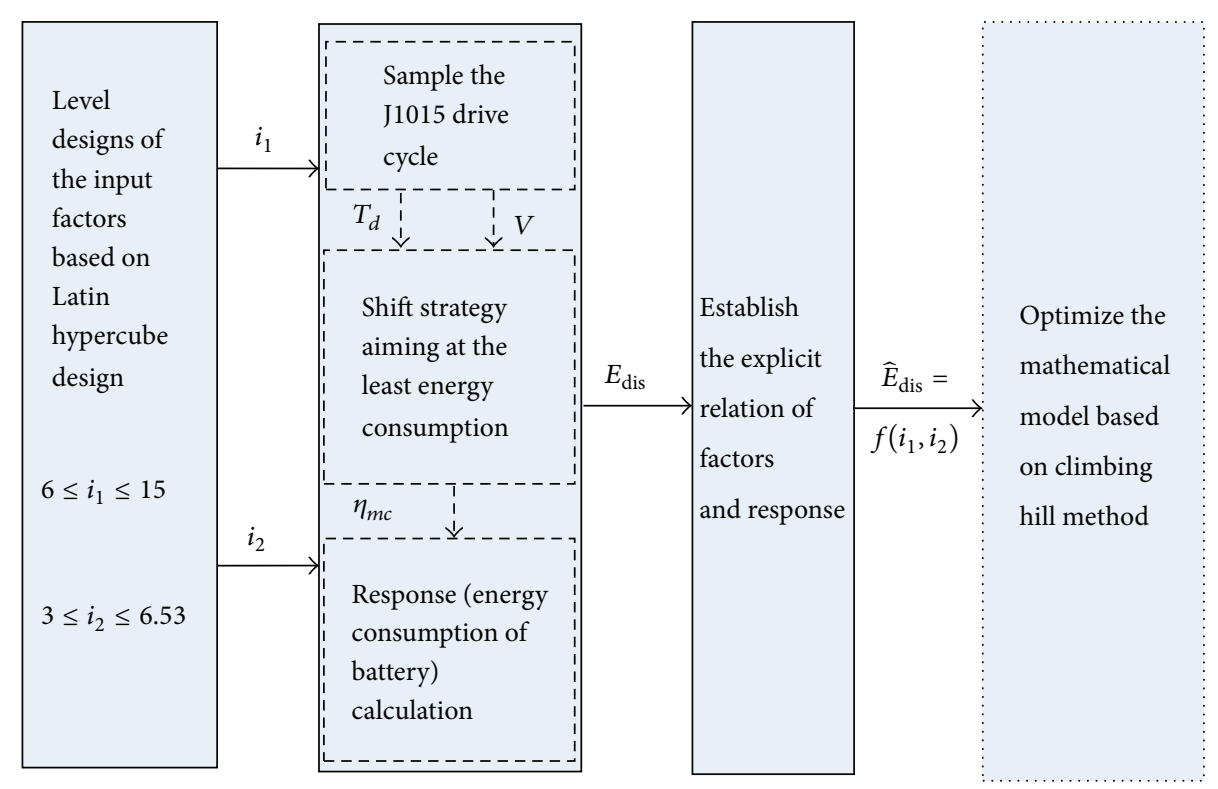

FIgURE 2: Route for establishing the mathematical model of energy consumption. $T_{d}$ : vehicle torque demand; $\eta_{m c}$ : motor efficiency.

where $\eta_{T}$ is the transfer efficiency of the mechanical drive system; $N_{1 \text { st_jk }}, T_{1 s t \_j k}, N_{2 \text { nd_jk }}$, and $T_{2 \text { nd_jk }}$ are motor speed and torque of the number $k$ LHD point in 1st and 2nd shift under number $j$ cycle point, which affect the drive efficiency of the small motor in 1st or 2nd shift as shown in (6) and Figure 3. Figure 3 shows the efficiency of the small power motor as function of speed and torque measured by motor test:

$$
\begin{aligned}
& \eta_{1 \text { st_ } j k}=f\left(N_{1 \text { st_ } j k}, T_{1 \text { st_jk }}\right), \\
& \eta_{2 \text { nd } j k}=f\left(N_{2 \text { nd_jk }_{-}}, T_{2 \text { nd } j k_{-}}\right) \text {, }
\end{aligned}
$$

where $\eta_{1 s t-j k}, \eta_{2 \text { nd_jk }}$ represent motor efficiency of the number $k$ LHD point in 1st and 2 nd shift under number $j$ cycle point.

Then, according to the peak performances of the two motors, which are the maximum speed of the small power motor $\left(N_{s \max }\right)$ and the pear torque of the high power motor $\left(T_{h \max }\right)$, the minimum energy consumption of the number $k$ LHD point under number $j$ drive condition can be obtained as shown in Figure 4. Penalty coefficient $\left(\Lambda_{j k}\right)$, a relatively small constant, is used when the motors are unable to function well under number $k$ LHD point. $\eta_{b j k}$ is the discharge efficiency of the battery relating to discharge current $\left(i_{\text {dis }}\right)$ and SOC, which has been measured by battery test as shown in Figure 5. Furthermore, the $i_{\text {dis }}$ and SOC can be obtained based on

$$
\begin{aligned}
i_{\text {dis }} & =\frac{T_{d j} V_{j}}{\max \left(\eta_{1 s t-j k}, \eta_{2 \text { nd } j k k}\right) U_{\mathrm{oc}}}, \\
\mathrm{SOC} & =\mathrm{SOC}_{0}-\frac{\int i_{\text {dis }} d t}{C_{\max }},
\end{aligned}
$$

where $\mathrm{SOC}_{0}$ is the initial state of SOC; $C_{\max }$ is the maximum capacity of the battery; $U_{\text {oc }}$ is the open circuit voltage of the battery.

Finally, the minimum energy consumption of the number $k$ LHD points under J1015 drive cycle is confirmed as shown in the following formula:

$$
E_{\text {disk }}=\sum_{j=1}^{N} E_{j k}
$$

where $N$ is the number of the sample points.

3.3. Mathematical Model for Energy Consumption. According to the processes mentioned above, the energy combustion 


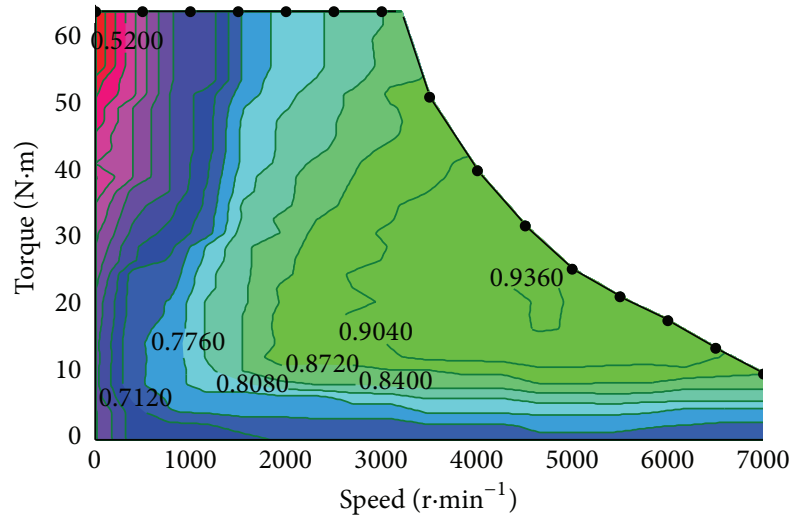

FIGURE 3: Efficiency of the small power motor as function of speed and torque.

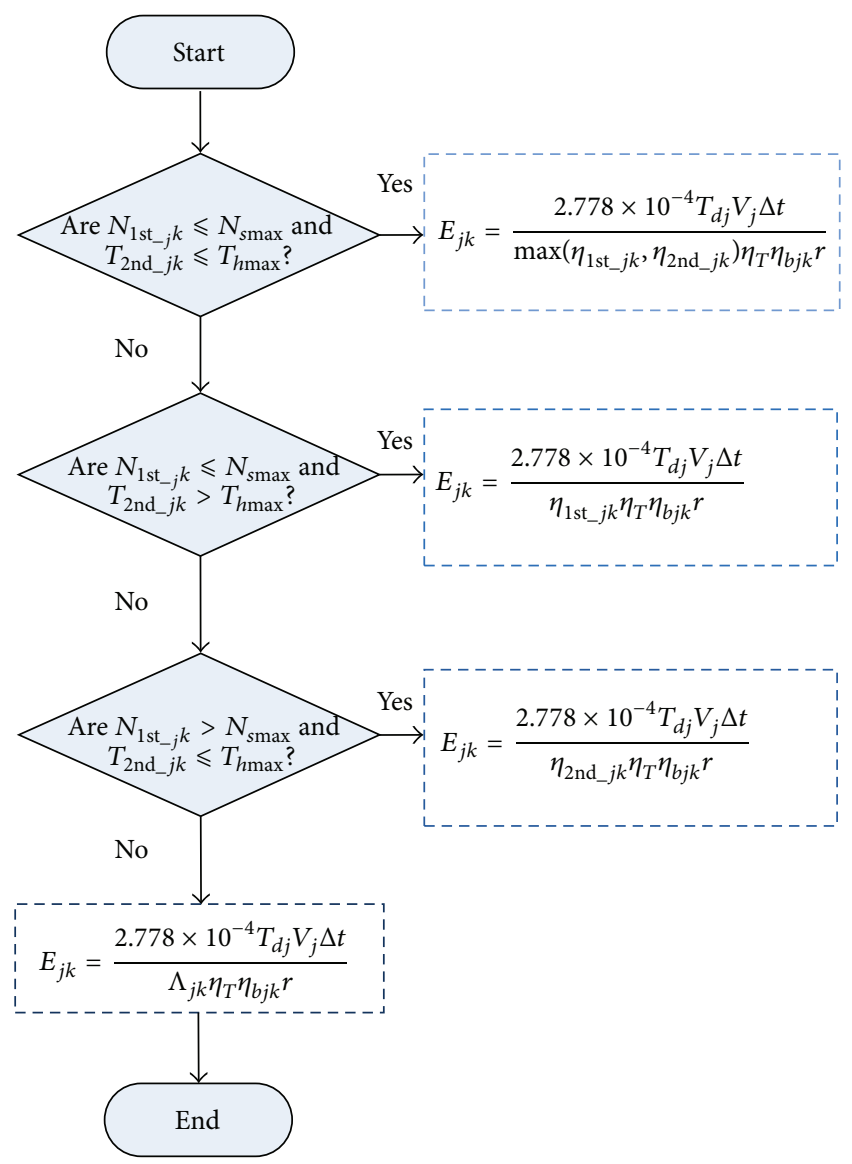

FIgURE 4: Stream for calculating energy consumption.

vector containing each LHD point can be determined as follows:

$$
\vec{Y}=\left[\begin{array}{c}
E_{\mathrm{dis} 1} \\
E_{\mathrm{dis} 2} \\
\vdots \\
E_{\mathrm{dis} m}
\end{array}\right] .
$$

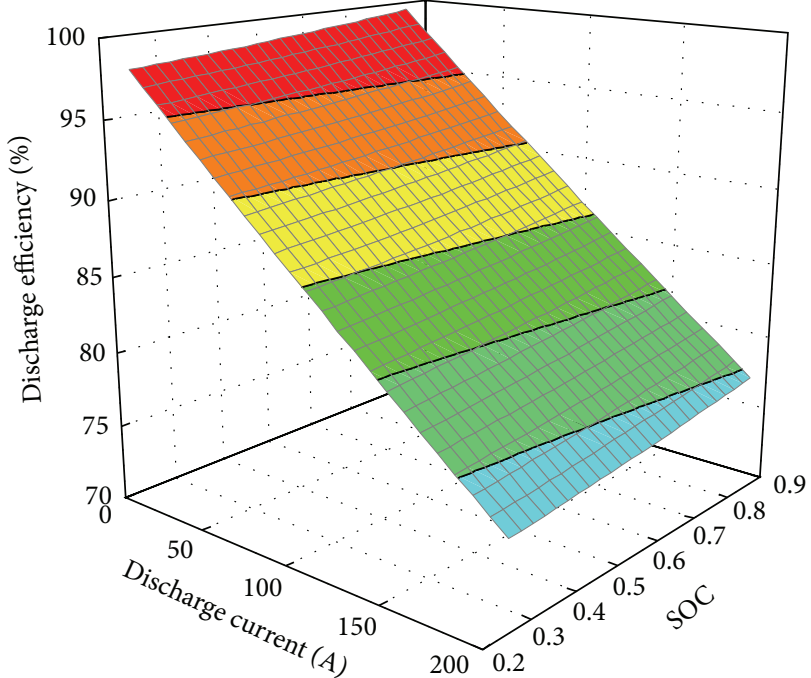

FIGURE 5: Efficiency of the battery as function of discharge current and SOC.

Suppose the mathematical model of the input factors $\left(i_{1}\right.$, $\left.i_{2}\right)$ and out response $\left(E_{\mathrm{dis}}\right)$ is a high-degree polynomial, taking sixth-degree polynomial as example as shown in formula (11). Currently, least squares method is most commonly used to obtain the formula coefficients but may have singular matrix problem. Furthermore, it is difficult to conduct significance test of the high-degree polynomial based on least squares method. Given the above analysis, a liner model is proposed as follows:

$$
\begin{gathered}
\hat{y}\left(\overrightarrow{x^{(k)}}, \vec{\alpha}\right)=\sum_{i=0}^{n-1} \alpha_{i} x_{i}, \\
x_{0}=1, \\
x_{1}=i_{1}, \\
x_{2}=i_{2}, \\
x_{3}=i_{1}{ }^{2}, \\
x_{4}=i_{2}{ }^{2}, \\
x_{5}=i_{1} i_{2}{ }^{2}, \\
x_{6}=i_{1}{ }^{3}, \\
x_{7}=i_{2}{ }^{3},
\end{gathered}
$$




$$
\begin{gathered}
x_{14}=i_{1}{ }^{3} i_{2}, \\
x_{15}=i_{1}{ }^{5}, \\
x_{16}=i_{2}{ }^{5}, \\
\vdots \\
x_{20}=i_{1}{ }^{4} i_{2}, \\
x_{21}=i_{1}{ }^{6}, \\
x_{22}=i_{2}{ }^{6}, \\
\vdots \\
x_{27}=i_{1}{ }^{5} i_{2},
\end{gathered}
$$

where $\hat{y}\left(\overrightarrow{x^{(k)}}, \vec{\alpha}\right)$ is the predictive energy consumption of the number $k$ LHD point; $\overrightarrow{x^{(k)}}$ is the vector of the number $k$ LHD point; $\alpha_{i}$ is the equation coefficients required to be solved according to the following optimization model:

$$
\begin{aligned}
\text { Find: } & \vec{\alpha} \in \overrightarrow{E^{k}} \\
\text { Minimize: } & \xi=\max \left|\widehat{y}\left(\overrightarrow{x^{(k)}}, \vec{\alpha}\right)-y^{(k)}\right| \\
\text { Satisfy: } & -\xi-\sum_{i=0}^{i=27} \alpha_{i} x_{i}^{(k)} \leq-y^{(k)} \\
& -\xi+\sum_{i=0}^{i=27} \alpha_{i} x_{i}^{(k)} \leq y^{(k)} \\
& \xi \geq 0,
\end{aligned}
$$

where $y^{(k)}$ is the actual energy consumption of the number $k$ LHD point.

In order to get a regression model in the form of standard liner program, (13) is utilized to substitute $\alpha_{i}$ as follows:

$$
\alpha_{i}=\beta_{i}-\lambda_{i}, \quad \beta_{i} \geq 0, \lambda_{i} \geq 0 .
$$

Bring the LHD points into the formulas and a standard liner program of the regression model is established as follows:

Find: $\quad \vec{\beta}, \vec{\lambda} \in \overrightarrow{E^{k}}$

Minimize: $\quad \xi=\max \left|\widehat{y}\left(\overrightarrow{x^{(k)}}, \vec{\alpha}\right)-y^{(k)}\right|$

Satisfy: $\quad \vec{A} \vec{U} \leq \overrightarrow{Y_{1}}$

$$
\vec{U} \geq 0
$$

where $\vec{U}, \vec{Y}_{1}$, and $\vec{A}$ are

$$
\begin{aligned}
& \vec{U}=\left[\begin{array}{llllll}
\xi & \beta_{0} & \beta_{1} \cdots \beta_{27} & \lambda_{0} & \lambda_{1} \cdots \lambda_{27}
\end{array}\right]^{\prime} \\
& \overrightarrow{Y_{1}}=\left[\begin{array}{ll}
-\vec{Y} & \vec{Y}
\end{array}\right]^{\prime} \\
& \vec{A}=\left[\begin{array}{ccc}
-\vec{I} & -\vec{X} & \vec{X} \\
-\vec{I} & \vec{X} & -\vec{X}
\end{array}\right] \\
& \vec{I}=\left[\begin{array}{lll}
1 & 1 & \cdots
\end{array}\right]^{\prime} \\
& \vec{X}=\left[\begin{array}{cc}
x_{0}^{(1)} & x_{1}{ }^{(1)} \cdots x_{27}^{(1)} \\
\vdots & \vdots \\
x_{0}{ }^{(N)} & x_{1}^{(N)} \cdots x_{27}^{(N)}
\end{array}\right] \text {. }
\end{aligned}
$$

As nonsignificant component in the mathematical model may cause adverse impact on the model complexity and illposed probability, progressive regression analysis is suggested to achieve the reduction of the model dimension [25]. Finally, the mathematical predictive energy consumption model, as function of the two-speed ratios, is obtained as shown in the following formula:

$$
\begin{aligned}
\widehat{E}_{\text {dis }}= & -1664.6817+697.12137 i_{1}+2135.7074 i_{2} \\
& -735.1532 i_{1} i_{2}-329.6006 i_{2}{ }^{2}-12.231346 i_{1}{ }^{3} \\
& +77.557368 i_{1}{ }^{2} i_{2}+129.0122 i_{1} i_{2}{ }^{2}+1.04928 i_{1}{ }^{4} \\
& -1.391006 i_{1}{ }^{3} i_{2}-18.18846 i_{1}{ }^{2} i_{2}{ }^{2} \\
& -0.028062 i_{1}{ }^{5}-0.106105 i_{1}{ }^{4} i_{2}+0.60974 i_{1}{ }^{3} i_{2}{ }^{2} \\
& +1.246804 i_{1}{ }^{2} i_{2}{ }^{3}-1.481546 i_{1} i_{2}{ }^{4} \\
& +0.676546 i_{2}{ }^{5}+0.0050322 i_{1}{ }^{5} i_{2} \\
& -0.0140566 i_{1}{ }^{4} i_{2}{ }^{2}-0.0660401 i_{1}{ }^{2} i_{2}{ }^{4} \\
& +0.12317 i_{1} i_{2}{ }^{5}-0.0695209 i_{2}{ }^{6}{ }^{6}
\end{aligned}
$$

\section{The Mathematical Model for Drive Strategy Optimization}

As shown in Figure 6, where HWFET drive cycle is utilized for vehicle test in highways, drive strategy of the DMVTEV varies with the vehicle working conditions obtained according to (4). In region I, the most important problem is to confirm the shift schedule of the rear power train system. Between region I and region II (region I-II), emphasis is placed on the switch law of SHMDM/SSMDM. As for region III, establishing a real-time and efficient power distribution model is the major concern. As the MEV with DMVT configuration rarely operates in region IV, simple power distribution based on settled rule aiming at vehicle dynamic performance is required. 


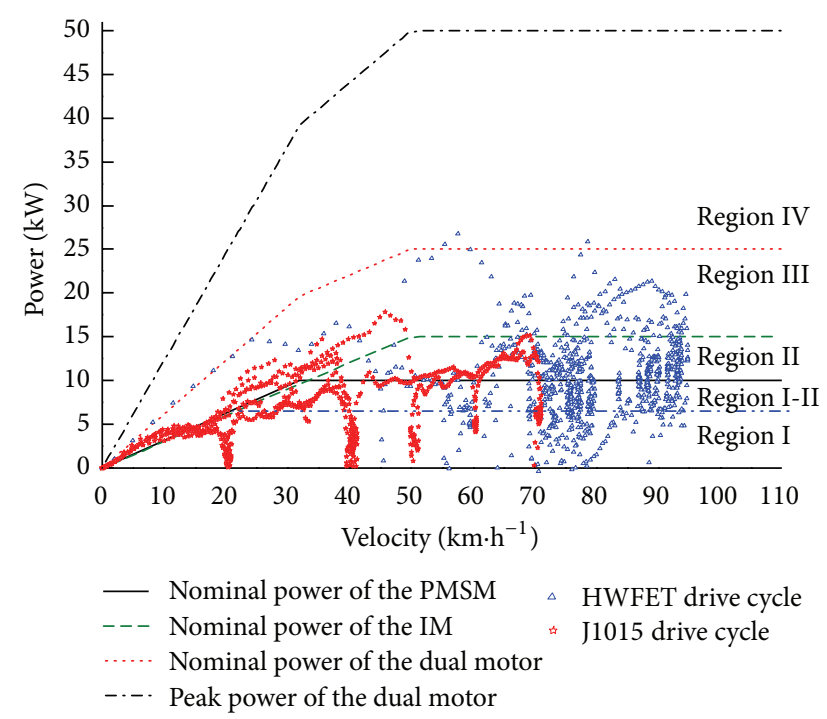

FIgURE 6: Power characteristics for the multiple drive modes as function of vehicle velocity.

4.1. Offline Optimization Process. As shown in Figure 7, given the above analysis, to develop an efficient and real-time drive strategy, an optimization program with multiple subroutines is proposed, which contains DOE, shift schedule optimization, switching law optimization, and power distribution optimization. Subroutines I and II are used to confirm the optimal shift and switch schedule, respectively. Based on offline GA, subroutine III is utilized to establish the optimal mathematical predictive model for power distribution.

4.2. Variables Design. The DOE points, which consist of $V(>0 \sim 110 \mathrm{~km} / \mathrm{h}), P_{d}$, and SOC $(0.25 \sim 0.85)$, are used to discrete the continuous space of the input factors. Using LHD method, the random characteristic of the vehicle drive conditions can be typified. To reduce the complexity of the optimization model, $P_{d}$ is used instead of $T_{d}$. Furthermore, as the peak discharge power of the battery may restrict the output power of the motors, correction of $P_{d}$ is required as shown in the following formula:

$$
\begin{aligned}
& P_{d \max } \\
& \quad=\min \left(P_{s \max }+P_{h \max }, P_{\text {dismax }}=M \times f(\mathrm{SOC})\right), \\
& P_{d c}=\min \left(P_{d}, P_{d \max }\right),
\end{aligned}
$$

where $P_{d \max }$ is the upper power limit of the total drive train system; $P_{s \max }$ and $P_{h \max }$ are the peak power of the small and high motor; $P_{\text {dismax }}$ is the maximum discharge power of the battery, which relates to SOC and can be confirmed based on the battery experiment results as shown in Figure $8 ; P_{d c}$ is the corrected power demand; $M$ is the quantity of the battery cells.

4.3. Problem Formulations of Each Region. For the three subroutines, we have the following.
(1) Subroutine I is used to confirm the shift schedule of the rear power train system with two-speed gearbox. First of all, according to formulas (4) (6), the number $i$ LHD points $V(i)$ and $P(i)$ can be converted to motor speed and torque in 1st and 2nd shift, and thus the motor efficiencies in 1st and 2nd shift can be obtained.

Then, aiming at the best drive efficiency while taking the peak performances of the two motors into consideration, the shift schedule can be expressed as follows:

(i) $\operatorname{Shift}(i)=1$ :

$$
\begin{aligned}
\text { If: } \eta_{1 \mathrm{st}}(i) & =\max \left(\eta_{1 \mathrm{st}}(i), \eta_{2 \mathrm{nd}}(i)\right), \\
N_{1 \mathrm{st}}(i) & \leqslant N_{s \max }(i) \\
P_{2 \mathrm{nd}}(i) & \leqslant P_{\text {smax }}(i)
\end{aligned}
$$

(ii) $\operatorname{Shift}(i)=1$ :

$$
\begin{gathered}
\text { If: } N_{1 s t}(i) \leqslant N_{s \max }(i), \\
P_{2 \mathrm{nd}}(i)>P_{s \max }(i) .
\end{gathered}
$$

(iii) $\operatorname{Shift}(i)=2$ :

$$
\text { If: } \begin{aligned}
\eta_{2 \mathrm{nd}}(i) & =\max \left(\eta_{1 \mathrm{st}}(i), \eta_{2 \mathrm{nd}}(i)\right), \\
N_{1 \mathrm{st}}(i) & \leqslant N_{s \max }(i) \\
P_{2 \mathrm{nd}}(i) & \leqslant P_{s \max }(i) .
\end{aligned}
$$

(iv) $\operatorname{Shift}(i)=2$ :

$$
\text { If: } \begin{aligned}
N_{1 s t}(i) & >N_{s \max }(i), \\
P_{2 \mathrm{nd}}(i) & \leqslant P_{s \max }(i),
\end{aligned}
$$

where $P_{2 n d}(i)$ is the power of small motor in 2nd shift.

(2) Based on subroutine II, the switching law of SHMDM/SSMDM can be obtained as follows.

Step 1. Call subroutine I to determine the optimal shift and then calculate the optimal battery power consumption of the number $i$ LHD point under SSMDM. The battery power consumption $P_{\text {dis_sm }}(i)$ is

$$
P_{\text {dis } \_m}(i)=\frac{\phi(i) P_{d c}(i)}{\eta_{s m}(i) \eta_{b}(i) \eta_{T}},
$$

where $\phi(i)$ is a penalty coefficient used to avoid the long-time overload operation of the small motor and thus prolong its service life, which can be expressed as

$$
\phi(i)=1+k \frac{P_{d c}(i) / \eta_{T}-P_{s e}(i)}{P_{s e}(i)},
$$

where $P_{s e}(i)$ is the nominal power of the small motor for the number $i$ LHD point and $k$ is a constant and bigger than 1 , which is regulated based on design requirement. 


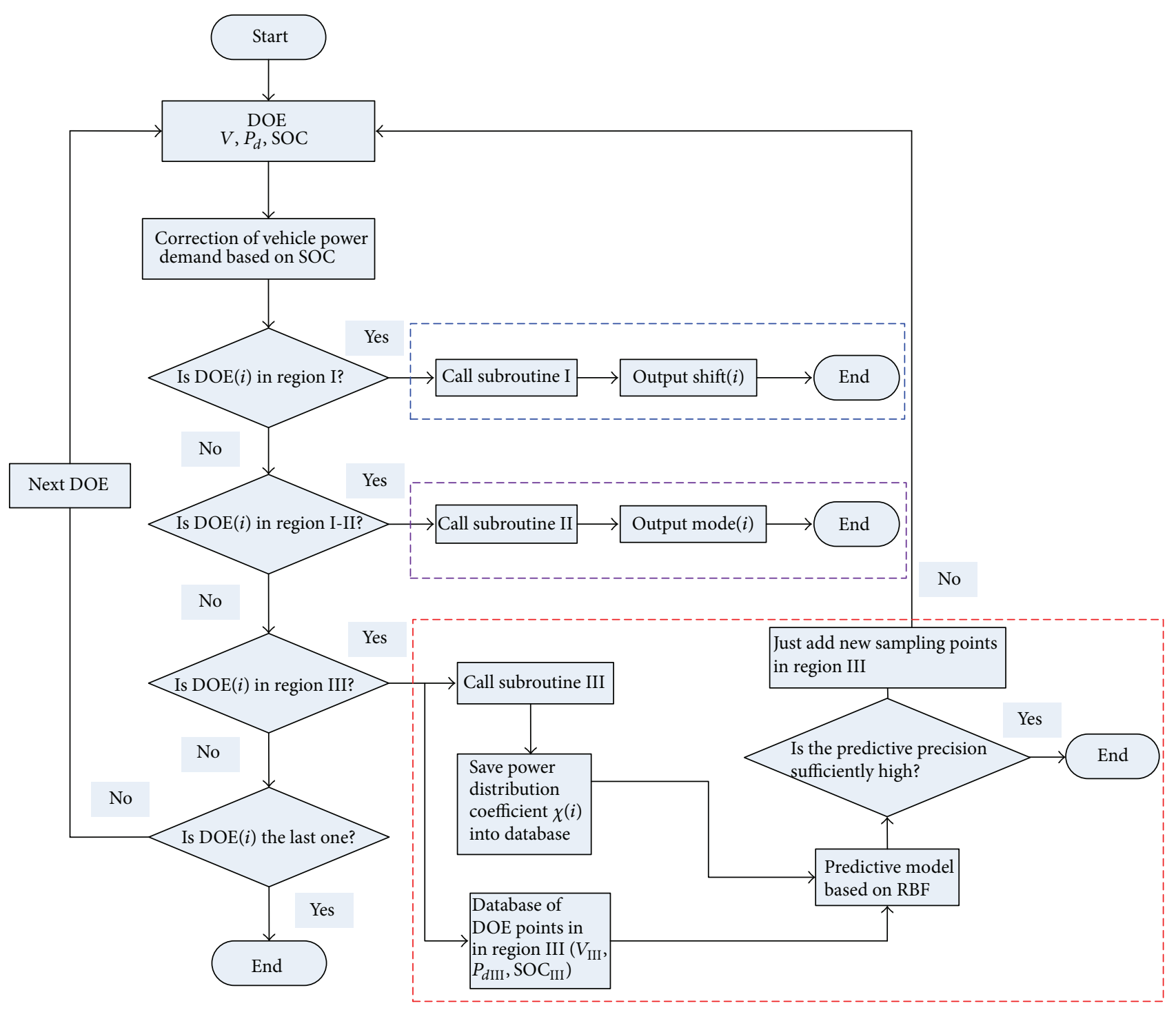

FIGURE 7: Offline optimization process for the drive strategy.

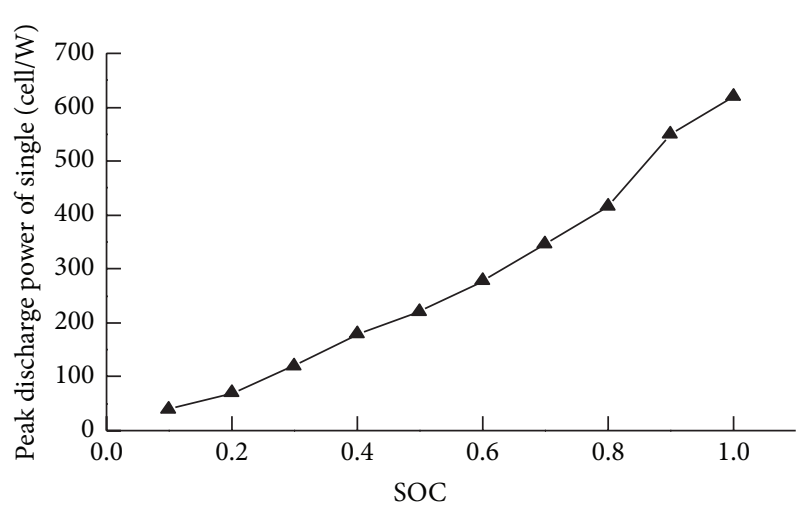

FIgURE 8: Peak discharge power of the single cell in the battery.

Step 2. Calculate the power consumption of the battery under SHMDM, then compare it with $P_{\text {dis_sm }}(i)$, and select the best one as the drive mode for the number $i$ LHD point, as shown in the following:

(I) $\operatorname{Mode}(i)=$ SSMDM:

If: $P_{\text {dis } s m}(i)$

$$
=\max \left(P_{\text {dis } \_s m}(i), P_{\text {dis } \_m}(i)=\frac{P_{d c}(i)}{\eta_{h m}(i) \eta_{b}(i) \eta_{T}}\right) .
$$

(II) $\operatorname{Mode}(i)=$ SHMDM:

$$
\text { If: } \begin{aligned}
& P_{\text {dis } \_h m}(i) \\
& =\max \left(P_{\text {dis } \_m}(i), P_{\text {dis } \_h m}(i)=\frac{P_{d c}(i)}{\eta_{h m}(i) \eta_{b}(i) \eta_{T}}\right),
\end{aligned}
$$

where $P_{\text {dis } h m}(i)$ is the battery power consumption of the number $i$ DOE point under SHMDM.

(3) To confirm the optimal power distribution coefficient $(\chi)$ between the two motors, which is the ratio of the power 
that distributed to the small motor to the corrected vehicle power demand, subroutine III is designed as follows.

Step 1. Establish the mathematical optimization model as shown in the following formula:

$$
\begin{aligned}
\text { Find: } & \chi=\frac{P_{s m}}{P_{d c}} \\
\text { Minimize: } & f(\chi)=\frac{\phi_{s} \chi P_{d c}}{\eta_{s m} \eta_{b} \eta_{T}}+\frac{\phi_{h}(1-\chi) P_{d c}}{\eta_{h m} \eta_{b} \eta_{T}} \\
\text { Satisfy: } \quad & \chi_{\max } P_{d c} \leq P_{s \max } \\
& \left(1-\chi_{\min }\right) P_{d c} \leq P_{h \max } \\
& N_{1 s t} \leq N_{s \max } \\
& 0 \leq \chi \leq 1,
\end{aligned}
$$

where $\phi_{s}, \phi_{h}$ are the penalty coefficients of the small and high power motor to prevent long-time overload operations, which can be expressed as follows:

$$
\phi_{s}= \begin{cases}1, & \chi P_{d c} \leqslant P_{s e} \\ 1+k_{s} \frac{\chi P_{d c} / \eta_{T}-P_{s e}}{P_{s e}}, & \chi P_{d c}>P_{s e}\end{cases}
$$$$
\phi_{h}
$$

$$
= \begin{cases}1, & (1-\chi) P_{d c} \leqslant P_{h e} \\ 1+k_{h} \frac{(1-\chi) P_{d c} / \eta_{T}-P_{h e}}{P_{h e}}, & (1-\chi) P_{d c}>P_{h e} .\end{cases}
$$

An optimization stream based on offline GA is designed to find the optimal distribution coefficients under the designed LHD points, as shown in Figure 9. Firstly, subroutine $\mathrm{I}$ is called to confirm the range of $\chi$; secondly, the first random population of $\chi$ is generated within $\chi_{\min }$ and $\chi_{\text {max }}$; thirdly, the fitness $f(\chi)$ of each individual in the population is evaluated according to the shift schedule and penalty coefficients; then, according to the fitness value, genetic-evolutionary process is repeated to generate a new population, which includes selection, crossover, and mutation; furthermore, the optimal $\chi$ for each LHD point can be confirmed by repeating the processes mentioned above; finally, save the optimal $\chi$ into the response database to establish the predictive model as shown in Step 2.

Step 2. Use the mathematical methods mentioned in Section 3.3 to set up a high-precision predictive model based on the factors database $\left(V, P_{d}, \mathrm{SOC}\right)$ and the optimal response database $(\chi)$. A feedback strategy, as shown in Figure 7, is designed to ensure the model precision. If the precision of the mathematical model is high enough, subroutine III will stop; if not, additional DOE points in region III will be generated.

\section{Results and Discussions}

5.1. Optimized Results of the Transmissions. For the mathematical predictive model of energy consumption as shown in formula (16), the multiple correlation coefficient $\left(R^{2}\right)$ of $\widehat{E}_{\text {dis }}$ is 0.998 and the studentized residuals are within -2 and +2 ; both indicate the mathematical model has a relatively high precision. Moreover, the predictive model is presented in the form of a high-degree geometric surface as shown in Figure 10, which clearly shows the geometrical convexity of the mathematical model. Therefore, hill-climbing searching method [26] instead of intelligent algorithm is utilized to optimize the transmissions. The optimal results of $i_{1}$ and $i_{2}$ are 9.905 and 4.828 , respectively.

In addition, the main and interactive effects of the input factors on energy consumption are presented in Figure 11. Generally, the main effect of $i_{1}$ on energy consumption becomes more significant as $i_{1}$ increases, while the main effect of $i_{2}$ on energy consumption becomes insignificant as $i_{2}$ increases, especially when $i_{2}$ is bigger than 12 . Furthermore, the interaction of $i_{1}$ and $i_{2}$ on energy consumption is significant, particularly when $i_{1}$ is smaller than 12 .

5.2. Optimized Results of the Shift and Switch Schedules. According to the optimized operations that are plotted on the velocity-power map under the various DOE points, the optimal shift and switch schedules are confirmed as shown in Figure 12. In order to eliminate the hunting problems of shift and switch, which are caused by the velocity lag behind the sudden change of pedal, shifting and switching delay strategies are adopted. Moreover, results show that, with the use of overload-penalty optimization strategy, the small power motor hardly operates under overload conditions in region I-II, which will be conducive to lengthening the service time of the small motor.

\subsection{Discussions of the Predictive Distribution Model}

5.3.1. Predictive Precision. First of all, a predictive model is established in the form of sixth-order polynomial. For this mathematical model, $R^{2}$ of $\hat{\chi}$ is 0.682 , which means the model has a very low predictive precision. Moreover, as shown in Figure 13(a), vertical cross sections of the polynomial at number 143 DOE point $\left(V=33.5845, P_{d}=19.2115\right.$, and SOC $=0.5879)$ are created and $95 \%$ confidence level is plotted on the figure simultaneously. Results show that most of the DOE points in the $P_{d}$-SOC, $V$-SOC, and $P_{d}-V$ cross sections are out of the $95 \%$ confidence level, which indicates that the mathematical model in the form of polynomial is incapable of characterizing the relationship of the optimal response $\chi$ and the input factors.

Given the above analysis, Gauss RBF is utilized to create the mathematical predictive model as shown in formula (28). For this model, $R^{2}$ of $\hat{\chi}$ is 0.989 . Furthermore, for the same vertical cross sections used in Figure 13(a), most of the predictive results of this model are within the $95 \%$ confidence interval as shown in Figure 13(b). Moreover, results also show that this regular pattern is also applicable to other vertical 


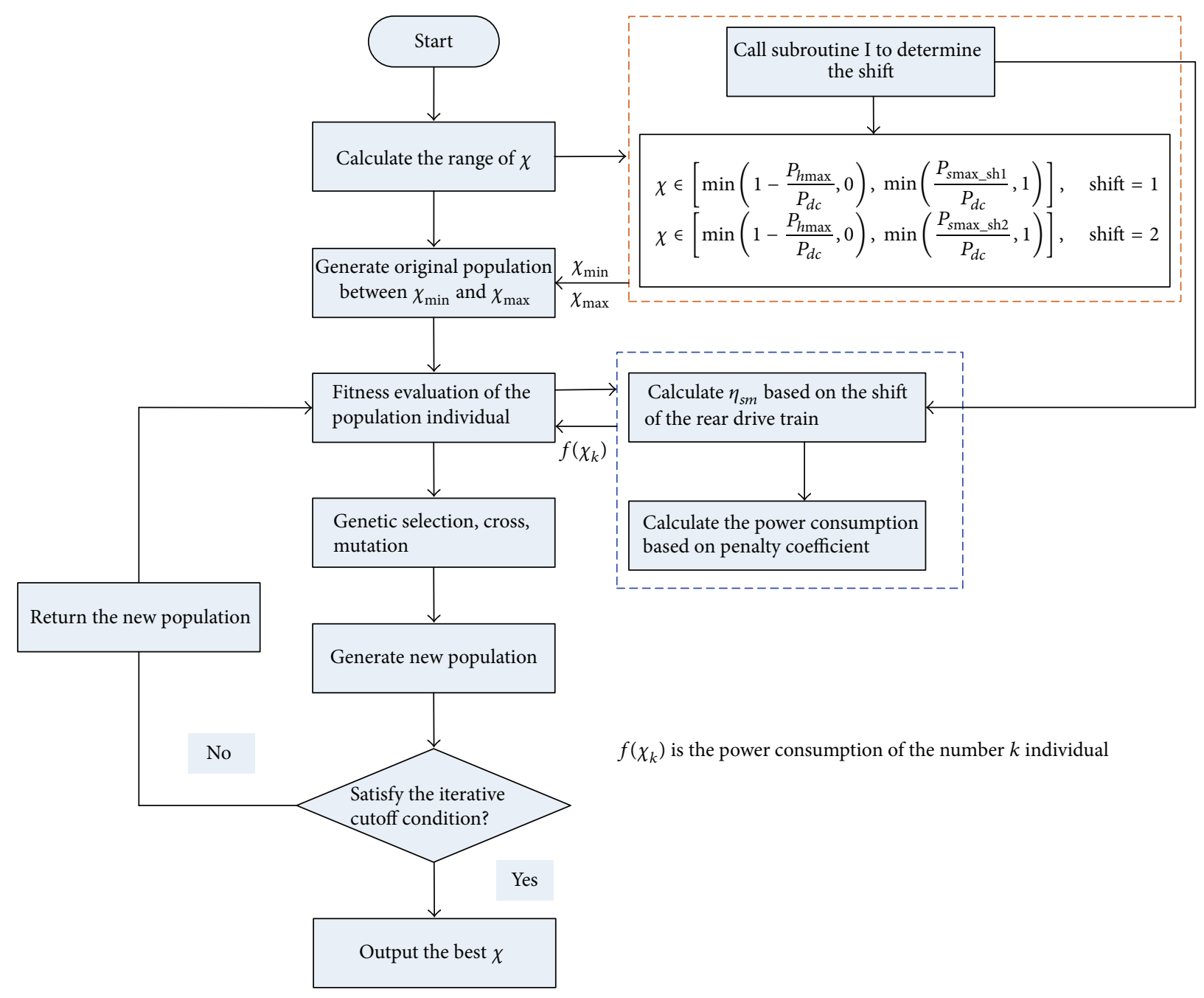

FIGURE 9: Offline optimization stream for the predictive model based on GA.

cross sections. Generally, the predictive precision of $\hat{\chi}$ based on Gauss RBF is high enough to achieve real-time power distribution:

$$
\begin{aligned}
\widehat{\chi}= & -305 h_{1}+28.35 h_{2}-292.8 h_{3}-36.34 h_{4} \\
& +92.09 h_{5}+77.16 h_{6}-11.54 h_{8}+20.39 h_{9} \\
& +834.9 h_{10}-452.2 h_{11}-6.7236 h_{12}+413.8 h_{13} \\
& -280 h_{15}+90.89 h_{16}-257.8 h_{17}-68.28 h_{18} \\
& +15.28 h_{19}+21.17 h_{20}+41.12 h_{21}+55.71 h_{23} \\
& -7.4086 h_{24}+26.98 h_{25} .
\end{aligned}
$$

In formula (28), $h_{i}$ is the Gauss basis function and can be expressed as in formula (29). In addition, the insignificant terms $\left(h_{7}, h_{14}\right.$, and $\left.h_{22}\right)$ are removed according to the progressive regression analysis:

$$
h_{i}=\exp \left(-\frac{\left\|\vec{X}-\vec{C}_{i}\right\|}{2 b_{i}^{2}}\right),
$$

where $b_{i}$ is set as a global width of 0.577 to reduce the difficulty while establishing the mathematical model; $\vec{C}_{i}$ is the RBF centre vector, which consists of 25 LHD points chosen according to the very low-precision regions in the polynomial model; $\vec{X}$ is the vector of the three factors in the dimensionless form as follows:

$$
\begin{aligned}
\vec{X} & =\left[\widetilde{V}, \widetilde{P_{d c}}, \widetilde{\mathrm{SOC}}\right]^{\prime} \\
\widetilde{V} & =\frac{V-\left(\left(V_{\max }-V_{\min }\right) / 2\right)}{\left(V_{\max }-V_{\min }\right) / 2} \\
\widetilde{P_{d c}} & =\frac{P_{d c}-\left(\left(P_{d \max }-P_{d \min }\right) / 2\right)}{\left(P_{d \max }-P_{d \min }\right) / 2} \\
\widetilde{\mathrm{SOC}} & =\frac{\mathrm{SOC}-\left(\left(\mathrm{SOC}_{\max }-\mathrm{SOC}_{\min }\right) / 2\right)}{\left(\mathrm{SOC}_{\max }-\mathrm{SOC}_{\min }\right) / 2} .
\end{aligned}
$$

5.3.2. Predictive Reliability. Although the mathematical model based on Gauss RBF has a highly predictive precision, predictive errors still exist. As shown in Figure 13(b), $\hat{\chi}$ may 


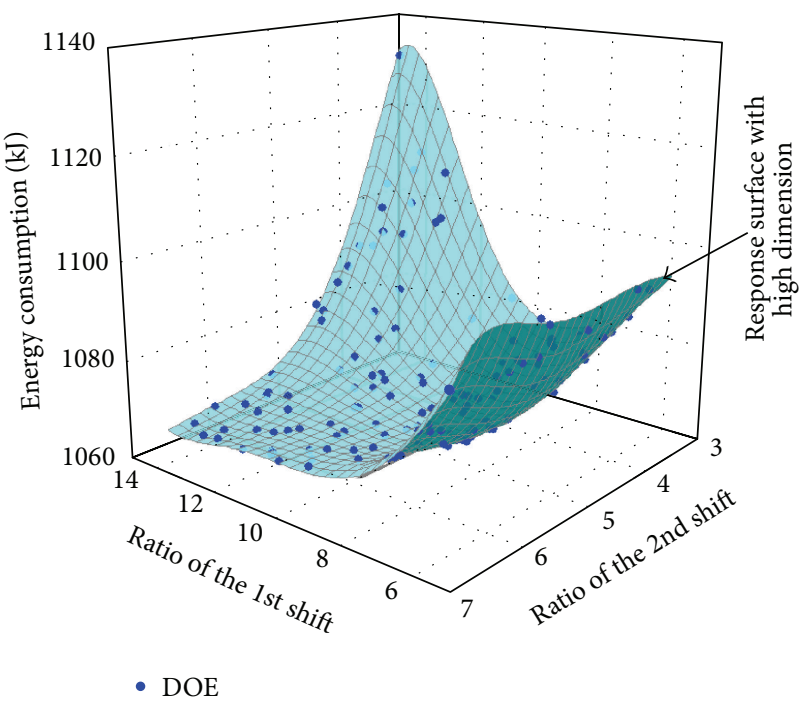

FIGURE 10: Response surface of the energy consumption.

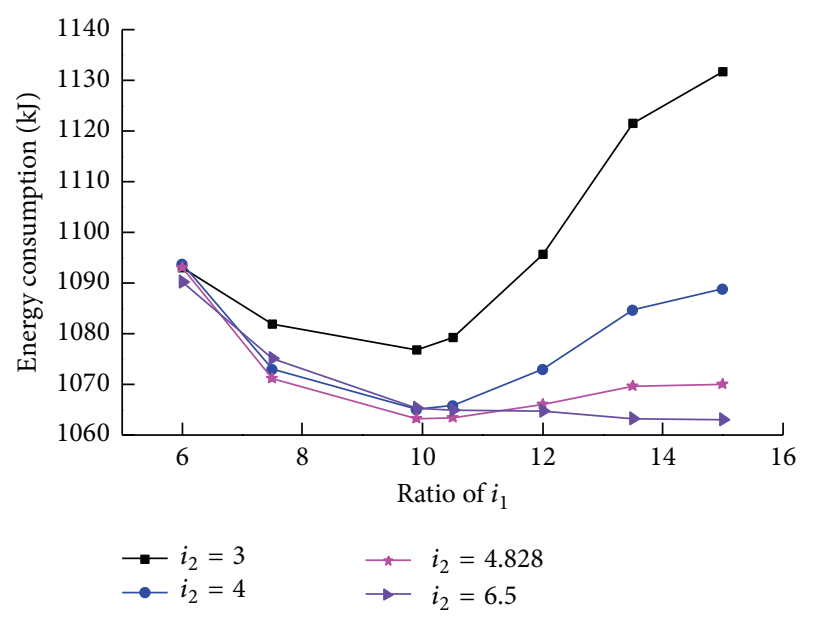

FIGURE 11: Effects of transmissions on energy consumption.

be out of the constraint between 0 and 1 (in some cases $\hat{\chi}_{\text {min }}$ may be bigger than 0 and $\hat{\chi}_{\max }$ may be smaller than 1), which will lead the predictive model falling into invalid solutions. To solve this problem, it is necessary to restrict the scope of $\hat{\chi}$ as follows.

When shift is in 1st,

$$
\begin{aligned}
\hat{\chi}_{\text {max }} P_{d c} & \leq P_{\text {smax } \_s h l}, \quad \hat{\chi}_{\max } \leq 1, \\
\left(1-\hat{\chi}_{\text {min }}\right) P_{d c} & \leq P_{h \text { max }}, \quad \hat{\chi}_{\text {min }} \geq 0 .
\end{aligned}
$$

When shift is in 2nd,

$$
\begin{aligned}
\hat{\chi}_{\text {max }} P_{d c} & \leq P_{\text {smax } \_s h 2}, \quad \hat{\chi}_{\max } \leq 1, \\
\left(1-\hat{\chi}_{\text {min }}\right) P_{d c} & \leq P_{h \max }, \quad \hat{\chi}_{\min } \geq 0,
\end{aligned}
$$

where $P_{s \max \_s h 1}, P_{s \max \_s h 2}$ are the maximum power of the small motor in 1st and 2nd shift.

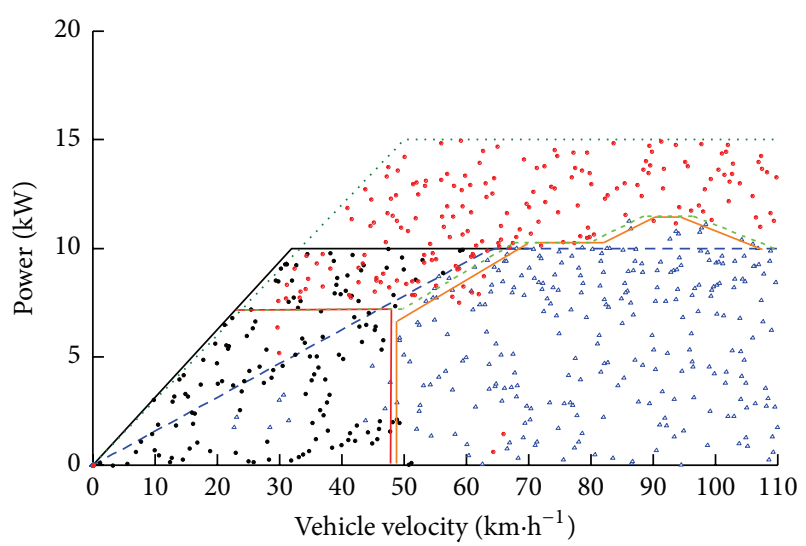
- Nominal power of the small motor in 1 st shift
- - - Nominal power of the small motor in $2 \mathrm{nd}$ shift
. Nominal power of the high power motor
- DOE in 1st shift
- DOE in 2nd shift
- DOE in SHMDM

FIGURE 12: Shift and switch schedules for the MEV.

TABLE 3: Economy results of the MEV driven by different power train configurations under unban cycle.

\begin{tabular}{lcc}
\hline \multirow{2}{*}{ Configuration } & \multicolumn{2}{c}{ J1015 drive cycle } \\
& Average efficiency & Energy consumption/kJ \\
\hline SMST & 0.7944 & 2265.31 \\
DMSG & 0.8183 & 2188.46 \\
DMVT & 0.8574 & 2094.2 \\
\hline
\end{tabular}

5.4. Simulation Verifications of the Optimized Scheme. As mentioned above, $P_{d}$ is adopted to make the optimization program much easier. But as for real-time control, $T_{d}$ is preferred, which can be transformed from $P_{d}$ based on formula (4). According to the optimal transmissions and drive strategy, as shown in Figure 14, the drive modes of the MEV under J1015 drive cycle are obtained in a real-time simulation model.

For the high power motor, most of the operations, marked in purple and black colours, are within the high-efficiency region under the SHMDM (mode 3 ) and the dual motor drive mode in 1st shift (mode 4). For the small motor, by using penalty algorithm, most of the operations in 1st shift (mode 1), 2nd shift (mode 2), and mode 4 lie in the nonoverload region, which are highlighted in blue, red, and murky red colour, respectively. Furthermore, the small motor also shows a good performance in operation efficiency.

Table 3 presents the economy performance of the MEV driven by different power train configurations. Compared with the SMST scheme, which is the original configuration of the MEV with a $25 \mathrm{~kW}$ (nominal power) PMSM and a settled reduction ratio $(i=6)$, the average drive efficiency of the optimized scheme reduces by $7.93 \%$ and energy consumption improved by $7.55 \%$ under J1015 drive cycle. In addition, compared with the traditional configuration with dual motor and settled gearbox (DMSG), which contains the same motors as in the DMVT scheme and fixed gear 


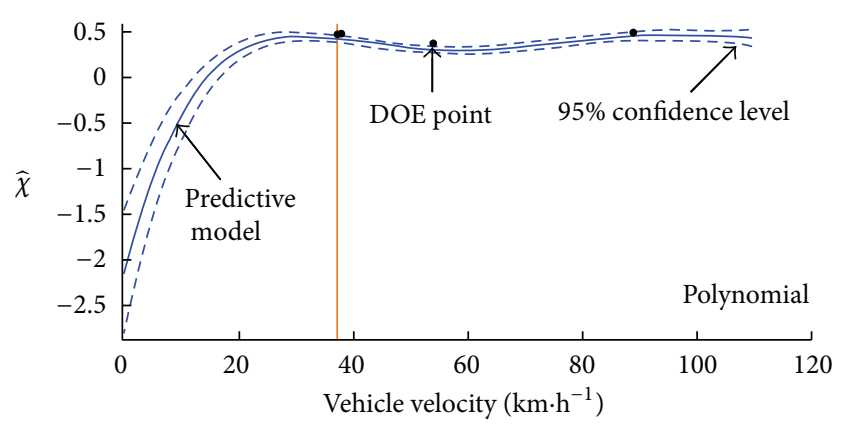

(A1) $P_{d}$-SOC vertical cross section

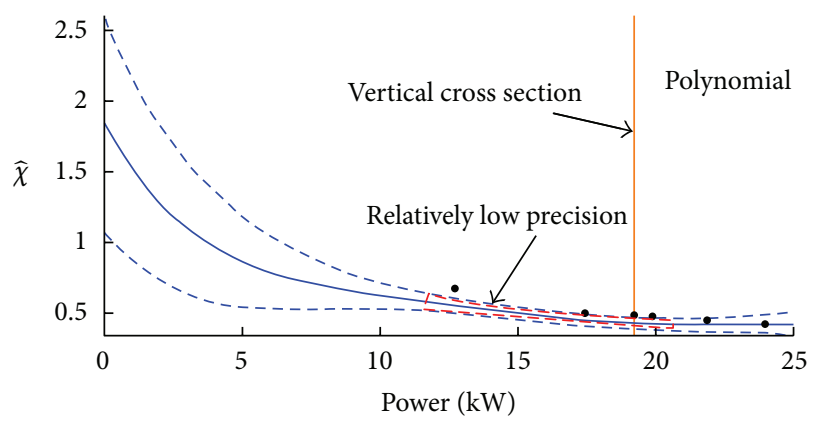

(B1) $V$-SOC vertical cross section

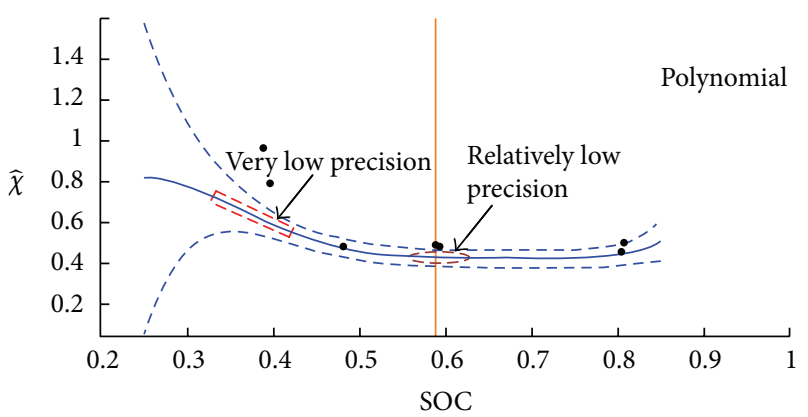

(C1) $P_{d}-V$ vertical cross section

(a)

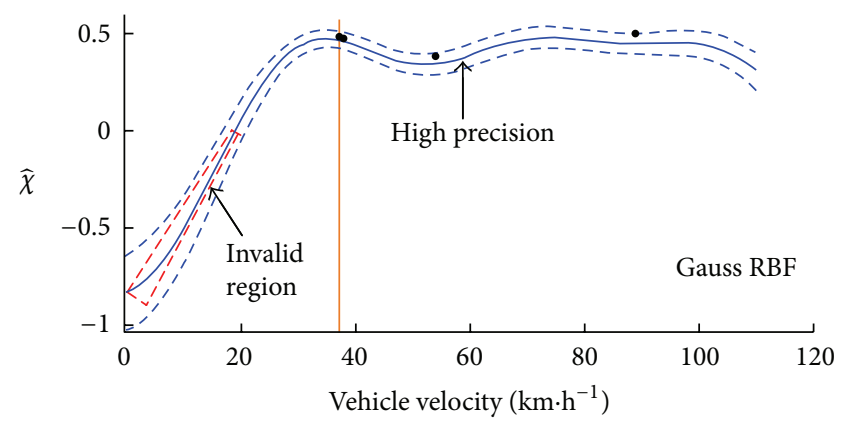

(A2) $P_{d}$-SOC vertical cross section

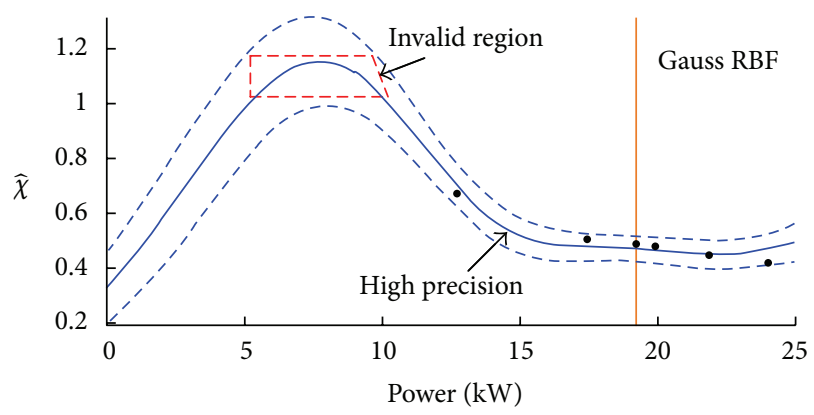

(B2) $V$-SOC vertical cross section

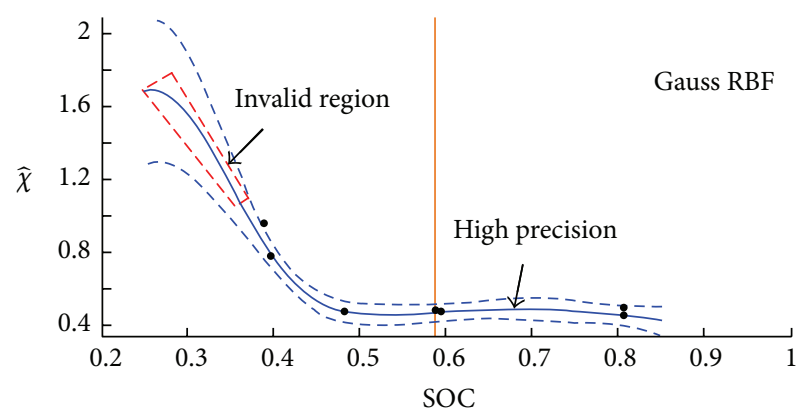

(C2) $P_{d}-V$ vertical cross section

(b)

FIGURE 13: (a) Predictive results based on polynomial. (b) Predictive results based on Gauss RBF.

ratios (one in front shaft is 5.82; the other in rear shaft is 6.5), the optimized two-speed scheme can improve energy consumption by $4.32 \%$.

Furthermore, as shown in Figure 15, the distribution of motor operations under J1015 drive cycle presents detailed comparisons between the SMSG and DMVT scheme. For the original drive scheme, most of the cycle operations, marked in red colour, deviate from the high-efficiency region. As for the DMVT configuration, the optimized power system and drive strategy improve the matching relationship of highefficiency region and motor operations highlighted in blue colour.

\section{Conclusions and Future Scope}

This paper presents detailed mathematical methods for economy optimization of a MEV with distributed power train system. First of all, the explicit functional relationship of energy consumption and transmissions is established with highly predictive precision, and the optimal solutions of the two-speed gear ratios are confirmed with the use of hillclimbing method: one is 9.905 and the other one is 4.828 . This mathematical method for ratio optimization can be applied to other types of multispeed transmission.

Then, to create an efficient and real-time drive strategy for the distributed power train system, an offline optimization program is proposed. The shift schedule for the two-speed gearbox and the switching boundaries between SSMDM and SHMDM are determined. A mathematical predictive power distribution model based on Gauss RBF is established, and the predictive precision and reliability of the model are verified. This power distribution method for dual-motor scheme can also be applied to multimotor configuration.

Finally, simulation verifications of the optimized scheme are carried out. Results show that, owing to the mathematical methods designed for the optimizations of transmissions 


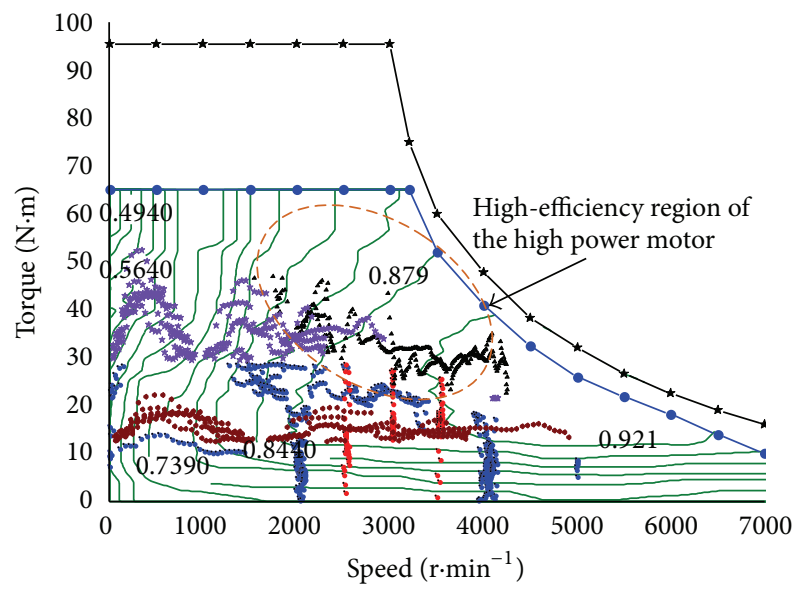
$\rightarrow$ Peak torque of the small power motor
* Peak torque of the high power motor
- Operations under mode 1
- Operations under mode 2
- Operations under mode 3
Operations of the small power motor under mode 4
* Operations of the high power motor under mode 4

FIGURE 14: Motor operation points of the DMVT scheme under J1015 drive cycle.

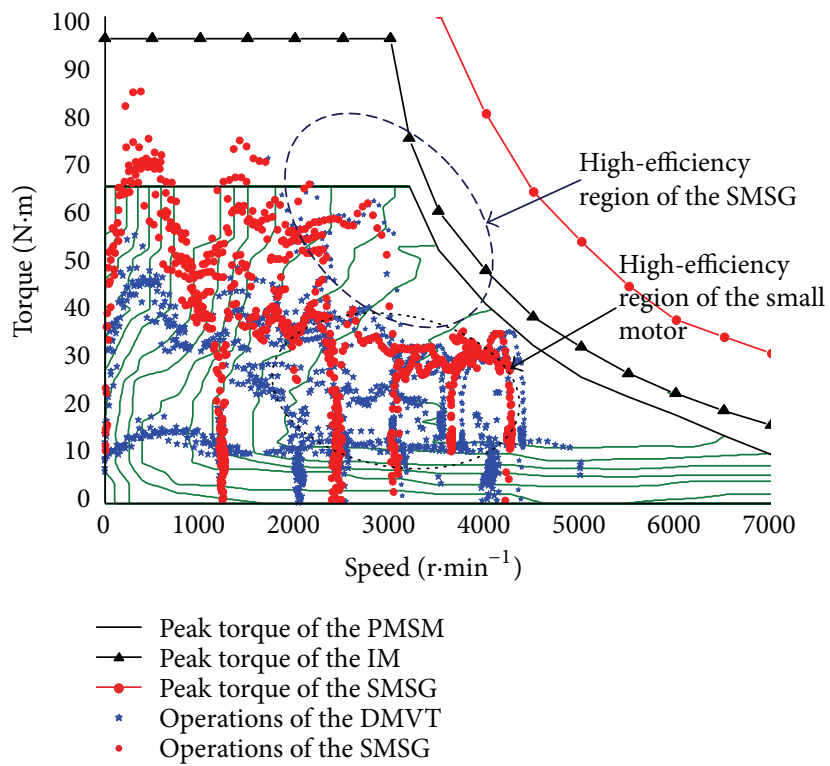

FIGURE 15: Motor operation points of the SMSG and DMVT schemes under J1015 drive cycle.

and drive strategy, the energy consumption of the DMVT configuration reduces by $7.55 \%$ and $4.32 \%$ when compared with the SMSG and DMSG scheme, respectively.

However, as a new power train configuration, further study is required to determine the optimal coordinated control strategy to reduce the switching jerk.

\section{Conflict of Interests}

The authors declare that there is no conflict of interests regarding the publication of this paper.

\section{Acknowledgments}

This work was supported by the Natural Science Foundation of Shandong Province, China (Grant nos. ZR2015EM054 and ZR2015EL025) and the Scientific and Technological Support Plan of Shandong Province, China (Grant no. 2015GGX105009).

\section{References}

[1] A. Santos, N. Mcguckin, and H. Nakamoto, Summary of Travel Trends: 2009 National Household Travel Survey, US Department of Transportantion, Washington, DC, USA, 2011.

[2] Ernst \& Young, Automotive Survey-Measuring Consumer Interest and Decision Criteria Regarding Plug-in Hybrid and Electric Vehicles in China, Ernst \& Young, Beijing, China, 2010.

[3] C. C. Chan, A. Bouscayrol, and K. Chen, "Electric, hybrid, and fuel-cell vehicles: architectures and modelling," IEEE Transactions on Vehicular Technology, vol. 59, no. 2, pp. 589-598, 2010.

[4] B. Gao, Q. Liang, Y. Xiang, L. Guo, and H. Chen, "Gear ratio optimization and shift control of 2-speed I-AMT in electric vehicle," Mechanical Systems and Signal Processing, vol. 50-51, pp. 615-631, 2015.

[5] Y. Hori, "Future vehicle driven by electricity and Controlresearch on four-wheel-motored 'UOT electric March II', IEEE Transactions on Industrial Electronics, vol. 51, no. 5, pp. 954-962, 2004.

[6] L. Xiong, F. M. Shi, S. L. Hu, and Y. Feng, "Development of a vehicle dynamics control system for four in-wheel motor EV," Applied Mechanics and Materials, vol. 427-429, pp. 1346-1349, 2013.

[7] Y. Luo and D. Tan, "Study on the dynamics of the in-wheel motor system," IEEE Transactions on Vehicular Technology, vol. 61, no. 8, pp. 3510-3518, 2012.

[8] Q. Ren, D. A. Crolla, and A. Morris, "Effect of transmission design on Electric Vehicle (EV) performance," in Proceedings of the 5th IEEE Vehicle Power and Propulsion Conference, pp. 12601265, IEEE, Dearborn, Mich. USA, September 2009.

[9] A. Morozov, K. Humphries, T. Zou, S. Martins, and J. Angeles, "Design and optimization of a drivetrain with two-speed transmission for electric delivery step van," in Proceedings of the IEEE International Electric Vehicle Conference (IEVC '14), pp. 18, Florence, Italy, December 2014.

[10] R. C. Sanghvi, A. S. Vashi, H. P. Patolia, and R. G. Jivani, "Multi-objective optimization of two-stage helical gear train using NSGA-II," Journal of Optimization, vol. 2014, pp. 1-8, 2014.

[11] V. Ngo, T. Hofman, M. Steinbuch, and A. Serrarens, "Predictive gear shift control for a parallel hybrid electric vehicle," in Proceedings of the 7th IEEE Vehicle Power and Propulsion Conference (VPPC '11), pp. 1-6, IEEE, Chicago, Ill, USA, September 2011.

[12] Z. Q. Tang, D. P. Ma, and X. W. Gong, "Transmission ratio optimization of two-speed pure electric vehicle," in Applied Mechanics and Materials, vol. 722, pp. 271-275, 2015.

[13] S. De Pinto, P. Camocardi, A. Sorniotti, G. Mantriota, P. Perlo, and F. Viotto, "A four-wheel-drive fully electric vehicle layout 
with two-speed transmissions," in Proceedings of the IEEE Vehicle Power and Propulsion Conference (VPPC '14), pp. 1-6, IEEE, Coimbra, Portugal, October 2014.

[14] TESLA, "Revolution of automobile engineering," 2015, https:// www.tesla.cn/models.

[15] N. Mutoh, "Driving and braking torque distribution methods for front- and rear-wheel-independent drive-type electric vehicles on roads with low friction coefficient," IEEE Transactions on Industrial Electronics, vol. 59, no. 10, pp. 3919-3933, 2012.

[16] N. Mutoh, T. Kato, and K. Murakami, "Front-and-rear-wheelindependent-drive-type electric vehicle (FRID EV) taking the lead for next generation ECO-vehicles," in Proceedings of the SAE 2011 World Congress, 2011-39-7, Detroit, Mich, USA, April 2011.

[17] H. Guo, H. He, and X. Xiao, "A predictive distribution model for cooperative braking system of an electric vehicle," Mathematical Problems in Engineering, vol. 2014, Article ID 828269, 11 pages, 2014.

[18] A. Sorniotti, T. Holdstock, G. L. Pilone et al., "Analysis and simulation of the gearshift methodology for a novel two-speed transmission system for electric powertrains with a central motor," Proceedings of the Institution of Mechanical Engineers Part D: Journal of Automobile Engineering, vol. 226, no. 7, pp. 915-929, 2012.

[19] L. Shaohua, D. Changqing, Y. Fuwu, W. Jun, L. Zheng, and L. Yuan, "A rule-based energy management strategy for a new BSG hybrid electric vehicle," in Proceedings of the 3rd Global Congress on Intelligent Systems (GCIS '12), pp. 209-212, IEEE, Wuhan, China, November 2012.

[20] M. Sorrentino, G. Rizzo, and I. Arsie, "Analysis of a rule-based control strategy for on-board energy management of series hybrid vehicles," Control Engineering Practice, vol. 19, no. 12, pp. 1433-1441, 2011.

[21] W. Yanzi, X. Changle, and W. Wang, "Energy management strategy based on fuzzy logic for a new hybrid battery-ultracapacitor energy storage system," in Proceedings of the IEEE Transportation Electrification Conference and Expo (ITEC AsiaPacific '14), pp. 1-5, IEEE, Beijing, China, September 2014.

[22] P. Pisu and G. Rizzoni, "A comparative study of supervisory control strategies for hybrid electric vehicles," IEEE Transactions on Control Systems Technology, vol. 15, no. 3, pp. 506-518, 2007.

[23] S. Stockar, V. Marano, M. Canova, G. Rizzoni, and L. Guzzella, "Energy-optimal control of plug-in hybrid electric vehicles for real-world driving cycles," IEEE Transactions on Vehicular Technology, vol. 60, no. 7, pp. 2949-2962, 2011.

[24] Q. Gong, Y. Li, and Z.-R. Peng, "Trip-based optimal power management of plug-in hybrid electric vehicles," IEEE Transactions on Vehicular Technology, vol. 57, no. 6, pp. 3393-3401, 2008.

[25] J. A. Rice, Mathematical Statistics and Data Analysis, China Machine Press, Beijing, China, 2011.

[26] L. Q. Yang, Numerical Analysis, Tsinghua University Press, Beijing, China, 2008. 


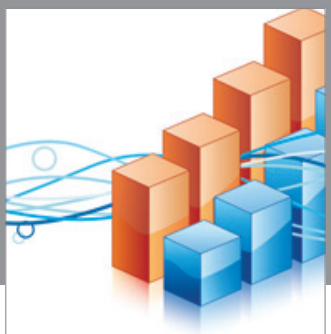

Advances in

Operations Research

vatem alat4

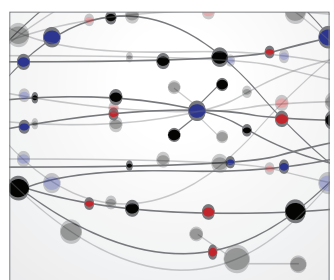

\section{The Scientific} World Journal
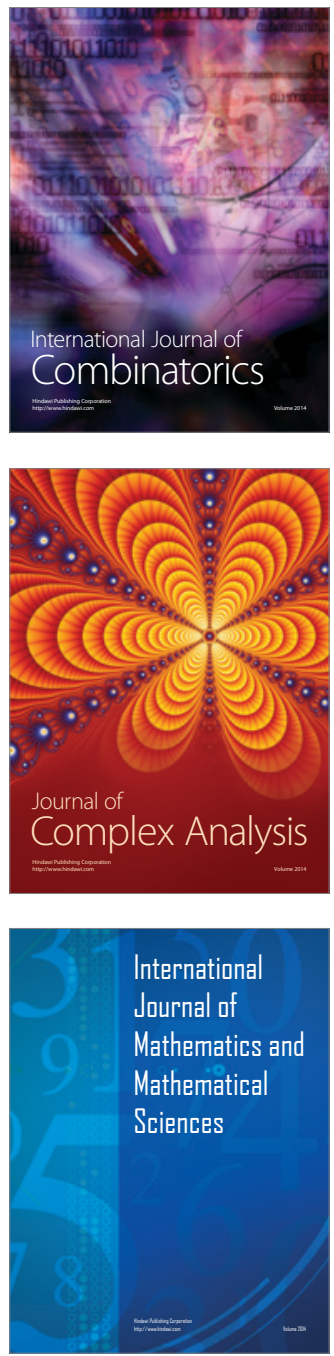
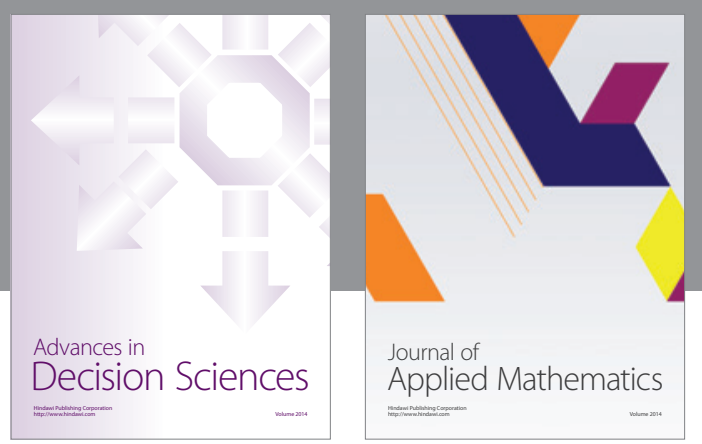

Algebra

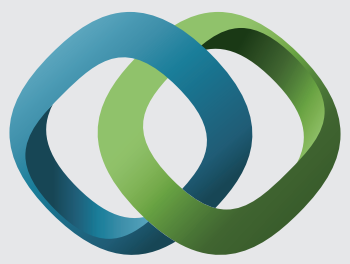

\section{Hindawi}

Submit your manuscripts at

http://www.hindawi.com
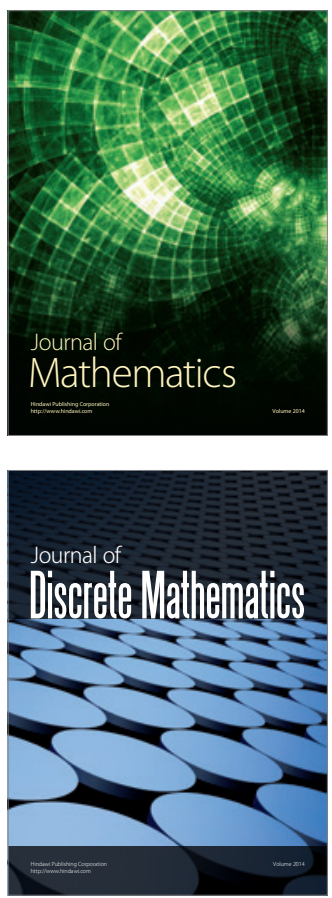

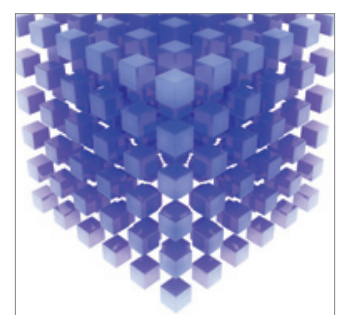

Mathematical Problems in Engineering
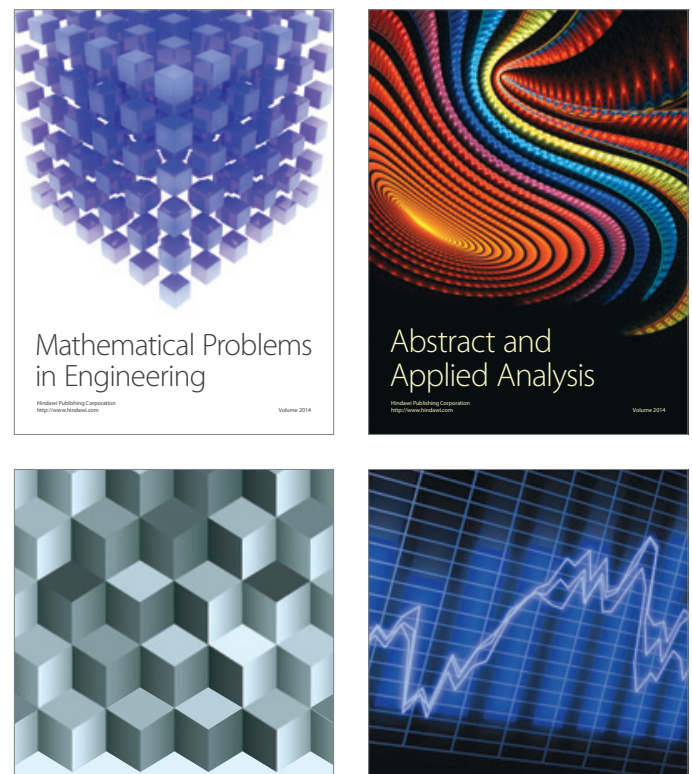

Journal of

Function Spaces

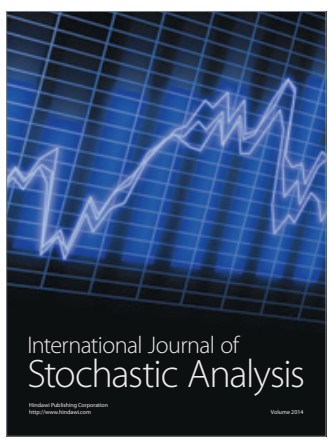

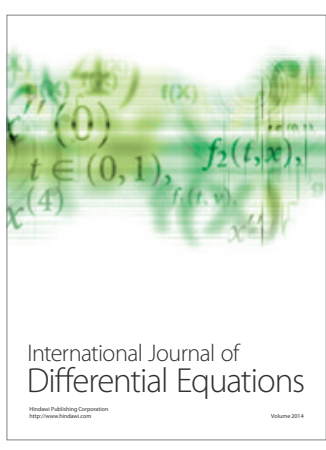
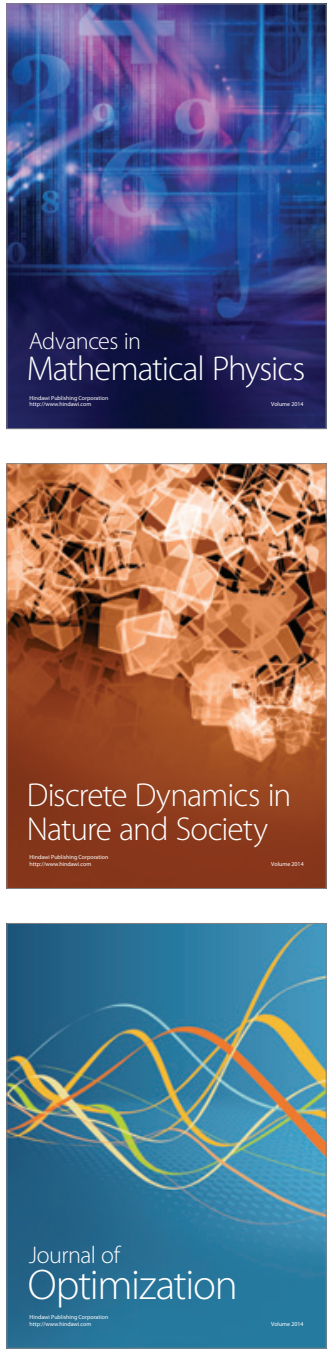